\title{
13.
}

\section{Lösung der Aufgaben $C$. und $D$. in Nr. 21. Band 45 dieses Journals S. 284.}

(Von dem Herrn Dr. phil. Lottner, Lehrer der Mathematik und Physik an der höhern Bürgerschule zu Lippstadt.)

\section{Aufgabe $C$.}

Es sei die aufzulösende Gleichung 4ten Grades, deren Coëfficienten, um die Brüche zu vermeiden, mit passenden Zahlen multiplicirt sind:

$$
x^{4}-8 a x^{3}-12 b x^{2}-48 c x+12 d=0 .
$$

Bezeichnet man :

$$
\left\{\begin{array}{c}
\sqrt{3}\left\{6 a^{2} d+18 c^{2}-b^{3}+3 b d+12 a b c\right. \\
\left.+\sqrt{ }\left[\left(6 a^{2} d+18 c^{2}-b^{3}+3 b d+12 a b c\right)^{2}-\left(b^{2}-8 a c+d\right)^{3}\right]\right\} \\
=\sqrt{ }\left[A+\sqrt{ }\left(A^{2}-B^{3}\right)\right] \text { mit } p \text { und } \\
3^{3}\left\{6 a^{2} d+18 c^{2}-b^{3}+3 b d+12 a b c\right. \\
\left.\left.-\sqrt{ }\left[6 a^{2} d+18 c^{2}-b^{3}+3 b d+12 a b c\right)^{2}-\left(b^{2}-8 a c+d\right)^{3}\right]\right\} \\
=\sqrt{3}\left[A-\sqrt{ }\left(A^{2}-B^{3}\right)\right] \text { mit } q,
\end{array}\right.
$$

(1.)

so sind, vorausgesetzt, dafs $A^{2}-B^{3}>0$ ist, die 4 Wurzeln der Gleichung:

(2.)

$$
\left\{\begin{aligned}
x_{1}= & 2 a+\sqrt{ }\left(4 a^{2}+2 b+p+q\right) \\
& +\sqrt{ }\left\{8 a^{2}+4 b-p-q+\sqrt{ }\left[\left(8 a^{2}+4 b-p-q\right)^{2}+3(p-q)^{2}\right]\right\} \\
x_{2}= & 2 a+\sqrt{ }\left(4 a^{2}+2 b+p+q\right) \\
& -\sqrt{ }\left\{8 a^{2}+4 b-p-q+\sqrt{ }\left[\left(8 a^{2}+4 b-p-q\right)^{2}+3(p-q)^{2}\right]\right\} \\
x_{3}= & 2 a-\sqrt{ }\left(4 a^{2}+2 b+p+q\right) \\
& +i \sqrt{ }\left(\left\{-8 a^{2}-4 b+p+q+\sqrt{ }\left[\left(8 a^{2}+4 b-p-q\right)^{2}+3(p-q)^{2}\right]\right\}\right. \\
x_{4}= & 2 a-\sqrt{ }\left(4 a^{2}+2 b+p+q\right) \\
& -i \sqrt{ }\left\{-8 a^{2}-4 b+p+q+\sqrt{ }\left[\left(8 a^{2}+4 b-p-q\right)^{2}+3(p-q)^{2}\right]\right\} .
\end{aligned}\right.
$$

Im andern Falle, wenn $A^{2}-B^{3}<0$ ist, setze man:

$$
\cos \alpha=\frac{6 a^{2} d+18 c^{2}+3 b d-b^{3}+12 a b c}{\left(b^{2}-8 a c+d\right)^{\frac{3}{2}}}=\frac{A}{B^{\frac{3}{2}}},
$$

so sind die vier Wurzeln: 
234 15. Lot tner, Lösung d. Aufgaben C. und D. in Nr.21. Bd.45 dies. Journ. S. 284.

(4.)

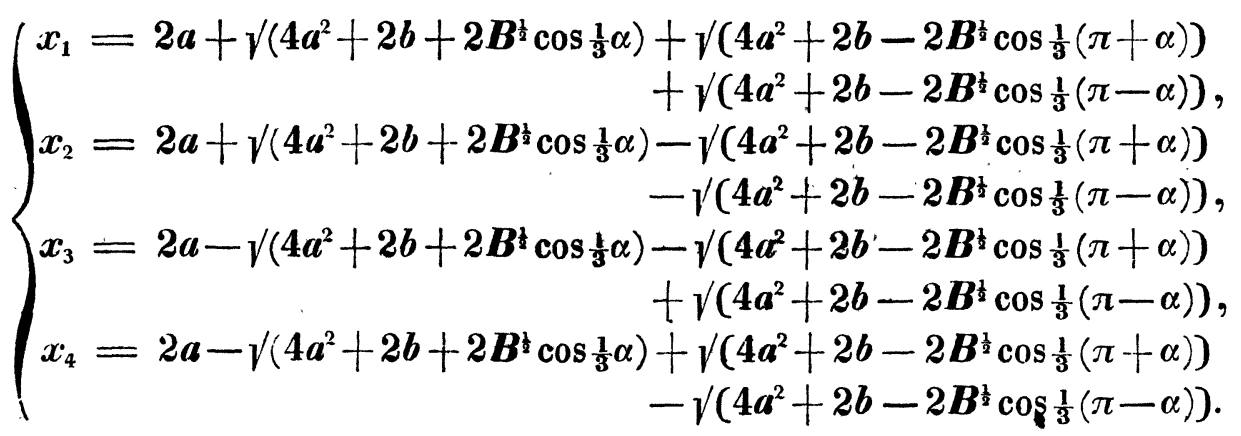

Wie man sieht, müssen in diesem Falle die vier Wurzeln entweder alle reell, oder alle imaginär sein, je nachdem $4 a^{2}+2 b-2 B^{\frac{1}{2}} \cos \frac{1}{3}(\pi+\alpha)$ und $4 a^{2}+2 b-2 B^{2} \cos \frac{1}{3}(\pi-\alpha)$ beide positiv oder einer von beiden Ausdrücken negativ ist.

\section{A ufgabe $D$.}

Es ist nun zu zeigen, dafs wenn $d=0$ ist, die Auflösung mit der der cubischen Gleichung

$$
x^{3}-8 a x^{2}-12 b x-48 c=0
$$

übereinstimmt.

Setzt man:

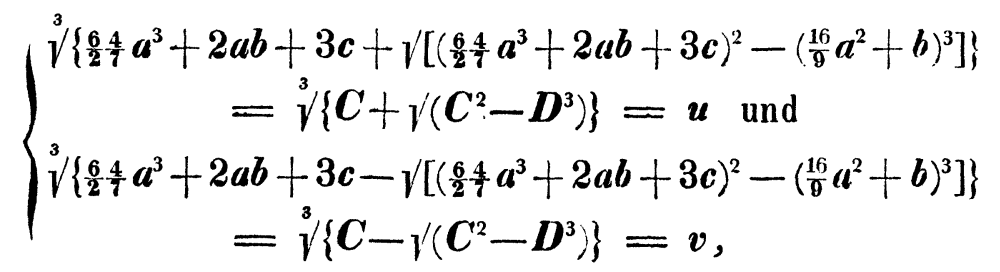

so sind bekanntlich die drei Wurzeln derselben:

(5 a.) $\quad x_{1}=\frac{8}{3} a+2(u+v), \quad x_{3}=\frac{8}{3} u-(u+v)-(u-v) \sqrt{ } 3 i$.

$$
\text { (6.) } \quad x_{2}=\frac{8}{3} a-(u+v)+(u-v) \sqrt{3} i \text {, }
$$

Wird $d=0$, so reduciren sich $p$ und $q$ auf:

$$
\text { (7.) }\left\{\begin{aligned}
p & =\sqrt[3]{ }\left[A+\sqrt{ }\left(A^{2}-B^{3}\right)\right] \\
& =\sqrt[3]{ }\left\{18 c^{2}+12 a b c-b^{3}+\sqrt{ }\left[\left(18 c^{2}+12 a b c-b^{3}\right)^{2}-\left(b^{2}-8 a c\right)^{3}\right]\right\} \\
y & =\sqrt[3]{ }\left[A-\sqrt{ }\left(A^{2}-B^{3}\right)\right] \\
& =\sqrt[3]{ }\left\{18 c^{2}+12 a b c-b^{3}-\sqrt{ }\left[\left(18 c^{2}+12 a b c-b^{3}\right)^{2}-\left(b^{2}-8 a c\right)^{3}\right]\right\} .
\end{aligned}\right.
$$

Es ist also zu zeigen, dafs nach der Substitution der Werthe (7.) in (2.), die Ausdrücke daselbst mit denen in ( $5 a$ a. $)$ übereinstimmen. 
15. Lot tner, Lösung d. Aufyaben C. und D. in Nr. 21. Bd.45 dies. Journ. S. 284.235

Man erhält

(8.) $\quad p=-\frac{4}{3} a v+u^{2}, \quad q=-\frac{4}{3} a u+v^{2}$.

Die Richtigkeit ergiebt sich aus folgender Rechnung.

Es müfste sein:

$$
\text { (9.) }\left\{\begin{array}{l}
p^{3}=u^{6}-4 a u^{4} v+\frac{16}{3} a^{2} u^{2} v^{2}-\frac{6}{2} \frac{4}{7} a^{3} v^{3} \\
q^{3}=v^{6}-4 a v^{4} u+\frac{16}{3} a^{2} u^{2} v^{2}-\frac{14}{2} \frac{4}{7} a^{3} u^{3}
\end{array}\right.
$$

oder, nach Einsetzung der Werthe aus (5. und 7):

$$
\text { (10.) }\left\{\begin{array}{c}
A+\sqrt{ }\left(A^{2}-B^{3}\right) \\
=2 C^{2}-D^{3}-4 a C D+\frac{16}{3} a^{2} D^{2}-\frac{6}{2} \frac{4}{7} a^{3} C+\left(2 C-4 a D+\frac{6}{2} \frac{4}{4} a^{3}\right) \sqrt{ }\left(C^{2}-D^{3}\right) \text { und } \\
A-\sqrt{ }\left(A^{2}-B^{3}\right) \\
=2 C^{2}-D^{3}-4 a C D+\frac{16}{3} a^{2} D^{2}-\frac{6}{2} a^{3} C-\left(2 C-4 a D+\frac{64}{2} a^{3}\right) \sqrt{ }\left(C^{2}-D^{3}\right) .
\end{array}\right.
$$

Hieraus müfste ferner folgen:

$$
\left\{\begin{aligned}
\left(2 C-4 a D+\frac{6}{2}+a^{3}\right)^{2}\left(C^{2}-D^{3}\right) & =A^{2}-B^{3} \\
2 C^{2}-D^{3}-4 a C D+\frac{16}{3} a^{2} D^{2}-\frac{6}{2} \frac{4}{7} a^{3} C & =A
\end{aligned}\right.
$$

Nun erhält man in der That, nach einiger Rechnung:

$$
\begin{gathered}
2 C-4 a D+\frac{6}{2} \frac{4}{7} a^{3}=2\left(\frac{6}{2} a^{3}+2 a b+3 c\right)-4 a\left(\frac{16}{7} a^{2}+b\right)+\frac{64}{2} \frac{4}{7} a^{3}=6 c, \\
A^{2}-B^{3}=3 b c^{2}\left(-b^{3}+9 c^{2}+12 a b c+\frac{128}{9} a^{3} c-\frac{4}{3} a^{2} b^{2}\right)=3 b c^{2}\left(C^{2}-D^{3}\right) .
\end{gathered}
$$

Die erste Gleichung (11.) wäre also verificirt; die zweite verwandelt sich, mit Hülfe der ersten, in:

(12.) $\left\{\begin{array}{l}\left(b c-\frac{128}{29} a^{3}\right) C+D^{2}\left(\frac{16}{3} a^{2}-D\right)=A, \text { oder in } \\ \left(b c-\frac{128}{29} a^{3}\right)\left(\frac{64}{27} a^{3}+2 a b c+3 c\right)+\left(\frac{16}{9} a^{2}+b\right)^{2}\left(\frac{32}{9} a^{2}-b\right)=12 a b c+18 c^{2}-b^{3} ;\end{array}\right.$

welche Gleichung nach einigen Reductionen sich ebenfalls als identisch erweiset. Da die beiden Gleichungen (11.) zur Bestimmung von $\boldsymbol{C}$ und $\boldsymbol{D}$ hinreichen und ihre Identität nachgewiesen ist, so folgt die Richtigkeit der Gleichungen (8.).

Es folgt aus denselben:

(13.)

$$
\left\{\begin{aligned}
p+q=-\frac{4}{3} a(u+v)+u^{2}+v^{2} & =\left(u+v-\frac{2}{3} a\right)^{2}-2 u v-\frac{4}{9} a^{2} \\
& =\left(u+v-\frac{2}{3} a\right)^{2}-2 D-\frac{4}{9} a^{2}, \\
p-q=\left(\frac{4}{3} a+u+v\right)(u-v) . &
\end{aligned}\right.
$$

Die Gröfse $4 a^{2}+2 b$ läfst sich unter der Form $\frac{4}{9} a^{2}+2 D$ darstellen; also ist:

$$
\begin{aligned}
p+q+4 a^{2}+2 b & =\left(u+v-\frac{2}{3} a\right)^{2}, \\
8 a^{2}-4 b-p-q & =\frac{8}{9} a^{2}+4 D+\frac{4}{3} a(u+v)-u^{2}-v^{2} .
\end{aligned}
$$

Crelle's Journal f. d. M. Bd. XLVII. Heft 3. 
236 13. Lottner, Lösung d. Aufgaben C. und D. in Nr.21. Bd.45 dies. Journ. S. 284.

Da $D=4 v$ ist, so wird hieraus :

(14.) $8 a^{2}-4 b-p-q=2\left[\left(\frac{4}{3} a+u+v\right)^{2}-3(u-v)^{2}\right]$,

folglich :

(15.)

$$
\begin{aligned}
& \left(8 a^{2}-4 b-p-q\right)^{2}+3(p-q)^{2} \\
& =\frac{1}{4}\left[\left(\frac{4}{3} a+u+v\right)^{2}-3(u-v)^{2}\right]^{2}+3\left(\frac{4}{3} a+u+v\right)^{2}(u-v)^{2} \\
& \left\{=\frac{1}{4}\left[\left(\frac{4}{3} u+u+v\right)^{2}+3(u-v)^{2}\right]^{2}\right. \text {, } \\
& \sqrt{ }\left(\left(8 a^{2}-4 b-p-q\right)^{2}+3(p-q)^{2}\right) \pm\left(8 a^{2}+4 b-p-q\right) \\
& =\left\{\begin{array}{l}
\left(\frac{4}{3}+u+v\right)^{2} \\
3(u-v)^{2}
\end{array}\right.
\end{aligned}
$$

Der erste Werth pafst für das obere, der zweite für das untere Zeichen.

Mit Hülfe dieser Transformationen verwandeln sich in dem Falle $d=0$ die Ausdrücke in (2.) in folgende:

$$
\begin{aligned}
& x_{1}=2 a+u+v-\frac{2}{3} a+\frac{4}{3} a+u+v=\frac{8}{3} a+2(u+v), \\
& x_{2}=2 a+u+v-\frac{2}{3} a-\frac{4}{3} a-u-v=0, \\
& x_{3}=2 a-u-v+\frac{2}{3} a-(u-v) \sqrt{3} \cdot i=\frac{8}{3} a-(u+v)-(u-v) \sqrt{ } 3 . i, \\
& x_{4}=2 a-u-v+\frac{2}{3} u+(u-v) \sqrt{3} . i=\frac{8}{3} a-(u+v)+(u-v) \sqrt{3} . i ;
\end{aligned}
$$

welche genau mit den Ausdrücken in (6.) übereinstimmen.

Für den Fall der trigonometrischen Auflösung verwandelt sich (3.) in

$$
\cos \alpha=\frac{A}{B^{\frac{3}{2}}}=\frac{18 c^{2}+12 a b-b^{3}}{\left(b^{2}-8 a c\right)^{\frac{3}{2}}} .
$$

Die dann entstehende cubische Gleichung hat, wenn

gesetzt wird,

$$
\cos \alpha_{1}=\frac{C}{D^{\frac{3}{2}}}=\frac{\frac{6}{2} \frac{4}{2} a^{3}+2 a b+3 c}{\left(\frac{1}{9}^{6} a^{2}+b\right)^{\frac{3}{2}}}
$$

$$
\text { (16.) }\left\{\begin{array}{l}
x_{1}=\frac{8}{3} a+4 D^{\frac{1}{2}} \cos \frac{1}{3} \alpha_{1}, \\
x_{2}=\frac{8}{3} a+4 D^{\frac{1}{3}} \cos \frac{1}{3}\left(\pi+\alpha_{1}\right), \\
x_{3}=\frac{8}{3} a+4 D^{\frac{1}{2}} \cos \frac{1}{3}\left(\pi-\alpha_{1}\right)
\end{array}\right.
$$

zu Wurzeln, deren Identität mit den Ausdrücken in (4.) für diesen Fall nachzuweisen ist.

Die Gleichungen:

$$
p=-\frac{4}{3} a v+u^{2}, \quad g=-\frac{4}{3} a u+v^{2}
$$

verwandeln sich jetzt in folgende:

$$
\left\{\begin{array}{l}
\boldsymbol{B}^{\frac{1}{2}} \boldsymbol{e}^{+\frac{1}{3} i \alpha}=-\frac{4}{3} \boldsymbol{a} \boldsymbol{D}^{\frac{1}{2}} e^{-\frac{1}{3} i \alpha_{1}}+\boldsymbol{D} \boldsymbol{e}^{+\frac{2}{3} i \alpha_{1}} \\
\boldsymbol{B}^{\frac{1}{2}} e^{-\frac{1}{3} i \alpha}=-\frac{4}{3} \boldsymbol{a} \boldsymbol{D}^{\frac{1}{2}} e^{+\frac{1}{3} i \alpha_{1}}+\boldsymbol{D} \boldsymbol{e}^{-\frac{2}{3} i \alpha_{1}}
\end{array}\right.
$$


15. Lot tu er, Lösung d. Aufgaben C. und D. in Nr.21. Bd.45 dies. Journ. S. 284. 237

Die Addition der Cuben dieser Ausdrücke giebt:

$$
B^{\frac{3}{2}} \cos \alpha=2 D^{3} \cos ^{2} \alpha_{1}-D^{3}-4 a D^{\frac{5}{2}} \cos \alpha_{1}+\frac{16}{3} a^{2} D^{2}-\frac{6}{2} \frac{4}{7} a D^{\frac{3}{2}} \cos \alpha_{1}
$$

oder

$$
A=2 C^{2}-D^{3}-4 a C D+\frac{19}{3} a^{2} D^{3}-\frac{6}{2} \frac{4}{7} a^{3} C ;
$$

also dieselbe Gleichung wie (11.).

Multiplicirt man die beiden Ausdrücke (17.), so erhält man für die zweite zur Bestimmung von $\boldsymbol{C}$ und $\boldsymbol{D}$ nothwendige Gleichung:

$$
\boldsymbol{B}=\frac{16}{9} a^{2} D-\frac{8}{3} a D^{\frac{3}{2}} \cos \alpha_{1}+D^{2}=\frac{16}{9} a^{2} D-\frac{8}{3} a C+D^{2},
$$

die ebenfalls durch eine kleine Rechnung als identisch sich erweiset. Also gelten die Ausdrücke in (17.).

Man erhält aus denselben leicht die reellen Formen:

$$
\left\{\begin{array}{l}
2 \boldsymbol{B}^{\frac{1}{2}} \cos \frac{1}{3} \alpha-\frac{8}{3} \boldsymbol{a} \boldsymbol{D}^{\frac{1}{2}} \cos \frac{1}{3} \alpha_{1}+2 \boldsymbol{D} \cos \frac{2}{3} \alpha_{1}, \\
2 \boldsymbol{B}^{\frac{1}{2}} \sin \frac{1}{3} \alpha_{1}-\frac{8}{3} \boldsymbol{a} \boldsymbol{D}^{\frac{1}{2}} \sin \frac{1}{3} \alpha_{1}+2 \boldsymbol{D} \sin \frac{2}{3} \alpha_{1} .
\end{array}\right.
$$

Berücksichtigt man, dafs $4 a^{2}+2 b=2 D+\frac{4}{9} a^{2}$ ist, so wird

$$
\text { (19.) } 4 a^{2}+2 b+2 B^{\frac{1}{2}} \cos \frac{1}{3} \alpha_{1}=\left(2 D^{\frac{1}{2}} \cos \frac{1}{3} \alpha_{1}-\frac{2}{3} a\right)^{2} \text {. }
$$

Ferner findet man

$$
\left\{\begin{array}{l}
2 \boldsymbol{B}^{\frac{1}{2}} \cos \frac{1}{3}(\pi \pm \alpha)=-\frac{8}{3} a D^{\frac{1}{2}} \cos \frac{1}{3}\left(\pi \mp \alpha_{1}\right)-2 D \cos \frac{2}{3}\left(\pi \mp \alpha_{1}\right) \\
4 a^{2}+2 b-2 B^{\frac{1}{2}} \cos \frac{1}{3}(\pi \pm \alpha)=\left(2 D^{\frac{1}{2}} \cos \frac{1}{3}\left(\pi \mp \alpha_{1}\right)+\frac{2}{3} a\right)^{2} .
\end{array}\right.
$$

Mit Hülfe von (19. und 20.) und der Formeln $\cos \frac{1}{3}\left(\pi-\alpha_{1}\right)+\cos \frac{1}{3}\left(\pi+\alpha_{1}\right)=\cos \frac{1}{3} \alpha_{1}, \quad \cos \frac{1}{3}\left(\pi-\alpha_{1}\right)-\cos \frac{1}{3}\left(\pi+\alpha_{1}\right)=\sqrt{3} \sin \frac{1}{3} \alpha_{1}$ verwandeln sich die Wurzeln (4.) in

$$
\begin{aligned}
& x_{1}=\frac{8}{3} a+4 D^{\frac{1}{2}} \cos \frac{1}{3} \alpha_{1}, \\
& x_{2}=0, \\
& x_{3}=\frac{8}{3} a-2 D^{\frac{1}{2}} \cos \frac{1}{3} \alpha_{1}-2 D^{\frac{1}{2}} / 3 \sin \frac{1}{3} \alpha_{1}=\frac{8}{3} a-4 D^{\frac{1}{2}} \cos \frac{1}{3}\left(\pi-\alpha_{1}\right), \\
& x_{4}=\frac{8}{3} a-2 D^{\frac{1}{3}} \cos \frac{1}{3} \alpha_{1}+2 D^{\frac{1}{2}} \sqrt{ } / 3 \sin \frac{1}{3} \alpha_{1}=\frac{8}{3} a-4 D^{\frac{1}{2}} \cos \frac{1}{3}\left(\pi+\alpha_{1}\right) .
\end{aligned}
$$

Dies sind die Ausdrücke (16.); mithin ist die in der Aufgabe verlangte Reduction in allen Fällen ausgeführt.

Lippstadt, den 19ten April 1853. 